\title{
Die öffentliche Soziologie und die Unmöglichkeit von Marienthal: $Z u$ einigen Preglau-Thesen
}

\section{Manfred Prisching}

Zusammenfassung: Anhand der klassischen Studie über Marienthal hat Max Preglau im Rahmen einer Ausstellungseröffnung vor wenigen Jahren einen Vortrag gehalten, in dem er die aktuelle Lage der Soziologie in Österreich erörtert. Seine Ausführungen werden in der vorliegenden Arbeit in Thesen gefasst und mit dem Blick auf die gegenwärtige Universitätsentwicklung diskutiert und weitergeführt.

Schlüsselwörter: Marienthal, Soziologie, öffentliche Wissenschaft, Universität, Projekte

\section{Public Sociology and the Impossibility of Marienthal. Some Preglau-Theses Revisited}

\begin{abstract}
When the opening of an exposition about the classical Marienthal-book was celebrated in 2008 Max Preglau used the opportunity for considering the topical situation of sociology in Austria. His considerations will be reformulated in several propositions, and by taking a look at the present development of the universities these propositions will be discussed and elaborated.
\end{abstract}

Keywords: Marienthal, sociology, public science, university, projects

M. Prisching $\square$

Universität Graz, Institut für Soziologie

Universitätsstraße 15/G4, 8010 Graz, Österreich

E-Mail: manfred.prisching@uni-graz.at 
Max Preglau hat im Jahr 2008 einen Vortrag, der aus Anlass der Eröffnung einer Ausstellung über die Marienthal-Studie (Jahoda, Lazarsfeld und Zeisel 1990) gehalten wurde und auf der Webpage des Grazer Archivs für die Geschichte der Soziologie Österreichs veröffentlicht ist (Preglau 2008), als Gelegenheit benutzt, über die aktuelle Situation der universitären Soziologie, insbesondere über ihren Bezug zu Politik und Öffentlichkeit, nachzudenken. Ich nehme seine Ausführungen zum Anlass, diese Thematik zu erörtern, da sie auch mit anderen seiner Interessen (von der kritischen Theorie über die Wissenschaftsforschung und den Besonderheiten einer postmodernen Politik bis zur allgemeinen Rolle der Soziologie) zusammenhängt.

Preglau stellt in seinem Vortrag Überlegungen darüber an, warum eine Studie wie jene über Marienthal eine derart nachhaltige Wirkung ausgeübt hat, dass sie sogar in den klassischen Kanon der Soziologie Eingang gefunden hat. Es ist seines Erachtens nicht nur die kreative Vielfalt der empirischen Methoden und die nüchterne Beobachtungsgabe der Forscher, sondern auch jene kritische Funktion der Sozialforschung, die gerade aus ihrer empirischen Vorurteilslosigkeit, aus ihrer empathischen Distanziertheit, aus ihrem sorgfältigen Hinschauen und Verstehen resultiert. Gerade dieser nüchterne Blick verschaffe jenen Menschen öffentliche Aufmerksamkeit und wenigstens symbolische Anerkennung, ,[... [ie in verbreiteter öffentlicher Ignoranz oder gar Verachtung, auf sich allein gestellt, ihre elende und verschämte Existenz zu fristen gezwungen waren $[\ldots]$ “, in einer Situation, in der die kapitalistische Durchdringung der Gesellschaft immer stärker zu dem zu führen begann, was Jürgen Habermas später als „Kolonialisierung der Lebenswelt“" (Habermas 1988) bezeichnen sollte. Preglau gesteht Studien wie der Marienthal-Studie sogar eine Impulswirkung in dem Sinne zu, dass sie zu einer sozialstaatlichen Zähmung kapitalistischer Dynamik geführt und die Alltagswelt der Bürgerinnen und Bürger vor überfordernden Anpassungszwängen geschützt haben. Als Beispiel für eine derartige Sichtbarmachung gesellschaftlicher Zwänge und Beengtheiten bleibt seines Erachtens die Marienthal-Studie bis heute relevant - und er schließt die Frage an, ob die Soziologie heute noch in der Lage wäre, eine solche Studie durchzuführen. Im Hinblick auf diese Frage ist er allerdings skeptisch, und er nennt einige Gründe für seine Skepsis, die ich in der Folge als seine „Thesen“ (wobei die Benennung dieser Thesen durch mich erfolgt) zitiere und diskutiere.

\section{Wissenschafts-Zerklüftungs-These}

Wir beginnen mit einem wissenschaftsimmanenten Problem: „Der Abstand zwischen den Disziplinen ist weiter fortgeschritten: Daher ist ein interdisziplinärer Zugang trotz vielfacher diesbezüglicher Postulate heute schwieriger geworden.“

Da hat Max Preglau wohl recht. Die Sozialwissenschaften, insbesondere auch die Soziologie, haben einen Ausweitungs- und Differenzierungsprozess hinter sich gebracht, der dazu führt, dass sich Sozialwissenschaftlerinnen und Sozialwissenschaftler mit großer Intensität 
ihren jeweiligen Spezialgebieten widmen. Nach der Mitte des 20. Jahrhunderts hat ein deutscher Soziologentag 6 bis 10 Vorträge aufgewiesen, die Protagonisten haben einander gekannt, alle Fachleute konnten lesen, was immer auf dem Gebiet der Soziologie publiziert worden ist, und tatsächlich waren sie auch über die benachbarten Disziplinen noch ziemlich gut informiert. Mittlerweile haben Soziologentage hunderte von Vorträgen im Programm; das aufgefächerte wissenschaftliche Repertoire wird an der Liste der Sektionen in den großen Fachgesellschaften und an der langen Liste spezieller wissenschaftlicher oder halbwissenschaftlicher Vereine sichtbar; selbst in den durch die Sektionen definierten Spezialgebieten erscheinen so viele Publikationen, dass man sie (auch wenn man sich auf Deutsch und Englisch beschränkt) nicht mehr lesen kann; und die Sektionen differenzieren sich weiter in Subsektionen, Arbeitsgemeinschaften oder andere der jeweiligen Abgrenzung dienliche Reviermarkierungen. Weitgehend wird dieser Expansions- und Differenzierungsprozess als „Professionalisierung“ oder „Normalisierung“ der Soziologie im Rahmen der Sozialwissenschaften begrüßt. Der Konkurrenzdruck wird stärker, die Karrieren werden unsicher, die ganze Situation unübersichtlich - da ist es allerdings auch von besonderer Bedeutung, wenn man seine (sei es auch nur vorgebliche) Originalität in immer weiter vorangetriebener Spezialisierungsemphase herausstreicht.

Das bedeutet zum ersten, dass die Relevanzkriterien sich verändern: Man muss seinen Platz behaupten, seine „Netzwerke“ und „Überlebensgemeinschaften“ enger knüpfen, seine Besonderheit inszenieren, in aktuelle Fragestellungen einsteigen - ganz unabhängig davon, ob die jeweiligen Fragen, Lösungen, Beschreibungen, Theorien oder Methoden nun an sich wichtig, brauchbar oder plausibel sind oder nicht. Wer bloß wissenschaftlich-intrinischen Motivationen folgt, hat nur in Ausnahmefällen eine Chance. Denn er muss ja auch an Themen und mit Methoden arbeiten, die sich in Projektanträgen und Evaluierungen bewähren - dabei ist er aber von den Gutachtern abhängig.

Es bedeutet zum zweiten, dass das Ganze der Gesellschaft aus dem Blick gerät: die großen Entwicklungslinien, die gegenwartsanalytischen Entwürfe, denen man aus der Sicht der jeweiligen thematischen oder methodischen Parzelle jeweils vorwerfen kann, allzu sehr über den Dingen zu schweben. Da man bekanntlich nur sehen kann, was man sehen kann, bedeutet der mit der Absteckung des Mini-Claims verbundene Aufwand aber auch, dass Kraft und Zeit nicht mehr aufgebracht werden können, sich weithin über soziologische Themen zu informieren die Soziologinnen und Soziologen werden im Laufe der Zeit so „ungebildet“, dass es ohnehin besser sein mag, wenn sie sich in ihren Nischen verkriechen, ohne in der Öffentlichkeit einen schlechten Eindruck zu hinterlassen. 


\section{Politik-Distanzierungs-These}

Die zweite Preglau-These betrifft die Außenbeziehungen der Soziologie: „Die Differenzierung zwischen Sozialwissenschaft und Politik hat zugenommen, das Interesse der WissenschafterInnen an politischen Problemen und das Interesse der Politik an Ergebnissen der Sozialwissenschaft ist geringer geworden."

Das ist eine Klage, die nicht neu ist. Schließlich hat sich die Soziologie erst nach dem Zweiten Weltkrieg soweit professionalisiert, dass sie der Politik und der Administration ihren Beitrag zum „Herrschaftswissen“ (Scheler 1968) andienen konnte. Die sechziger und siebziger Jahre waren dabei besonders ambivalent. Auf der einen Seite waren es die Jahre einer technokratischen Überschätzung - die Soziologinnen und Soziologen waren der Überzeugung, dass es nur noch einiger Studien bedürfe, um auf dem Wege zur Optimierung der Gesellschaft tüchtig voranzukommen (Schelsky 1979). Auf der anderen Seite waren es die Jahre einer marxistischen Pandemie, die - ganz im Gegensatz zur technokratischen Version - die Verderbnis der bestehenden kapitalistischen Gesellschaft in den düstersten Farben malte und eher auf die große Verweigerung statt auf konstruktiv-reformerische Mitwirkung abzielte.

Beide Zugänge haben sich im Laufe der Jahre in ihrer Überzeugungskraft relativiert und in ihrer Wirksamkeit reduziert. Die technokratische Hybris ist dahingeschmolzen, trotz mancher Besserwisserei aus sozialwissenschaftlichen Revieren. Die Attraktivität marxistischer Argumente ist beinahe vollständig verschwunden, zusammen mit dem Untergang des bolschewistischen Imperiums. Es bleibt das reformistische Anliegen, zu dem die Soziologie nach Ansicht ihrer Akteure durchaus manche Beiträge leisten könnte. Aber in dieser Hinsicht hat sich in der Tat ein „Normalisierungsprozess“" vollzogen: Für eine politische Visionendiskussion (kritische Gesellschaftsanalyse), aber auch für die administrative Machbarkeitsebene (Bereitstellung von Lösungen) mag die Soziologie weniger bedeutsam geworden sein, in den allgemeinen Projekten und Prozessen ist sie als Fach jedoch viel weniger umstritten als noch vor einigen Jahrzehnten. Es gehört beispielsweise eher schon zum Handwerkszeug, dass bei technischen Projekten aus dem IT-Bereich, dem Stadt- oder dem Architekturbereich eine „sozialwissenschaftliche Begleitung“ zu erfolgen hat; es gehört ebenso zum normalen Instrumentarium jedes Managements, von Zeit zu Zeit eine Kundenbefragung durchzuführen, ob es sich nun um die städtischen Verkehrsbetriebe oder um die Landeskrankenhäuser handelt; und bei diversen öffentlichen Ereignissen werden auch Soziologinnen und Soziologen um Interviews gebeten, ohne dass man sie von vornherein in der Rolle vermutet, eine grundsätzliche Antisystem-Haltung einzunehmen (Politikwissenschaftler geben ja ebenfalls ständig ihre Kommentare zu politischen Events oder Wahlen ab.) „Normalisierung“ dieser Art bedeutet aber auch eine Abnahme des Spektakulären. Soziologie ist nicht mehr aufsehenerregend, aber das hat auch sein Gutes. Die Normalisierung mag von manchen als eine Art von Arrangement mit den Machthabern der bestehenden Ord- 
nung angesehen werden, aber man kann nicht immer beides haben: konkrete Wirksamkeit und konsequente Antihaltung.

\section{Drittmittel-Dominanz-These}

Die dritte Preglau-These bezieht sich auf eine voranschreitende Neuordnung des gesamten akademischen Bereichs: „Verstärkte Drittmittelabhängigkeit der Forschung bindet Forschung stärker an die privaten Interessen finanzkräftiger Auftraggeber oder an die rein innerwissenschaftlichen Qualitäts- und Relevanzkriterien der öffentlichen Fördergeber.“

In der Tat schreitet die Neudefinition dessen, was eine „Universität“ sein soll, voran. Die „Selbstverständlichkeiten“ sind im öffentlichen Diskurs verändert worden. Früher ging es in der Wissenschaft um Erkenntnis, Wahrheit, Fortschritt, Aufklärung, auch wenn die Wichtigkeit von Fortschritten in der Medizin betont und ein paar neue elektronische Geräte positiv geschätzt wurden. Wenn heute von „neuen Erkenntnissen“ die Rede ist, dann ist normalerweise der Beitrag der Wissenschaft zu „,neuen Produkten“ gemeint. Wenn man vom „Fortschritt“ spricht, dann ist damit jenes wirtschaftliche Wachstum gemeint, welches nur durch wissenschaftsinduzierte Innovationsdynamik erzielt werden kann. Diese äußere Bindung der wesentlichen Wissenschaftsbereiche an die wirtschaftliche Entwicklung entspricht zugleich einer Neugestaltung der Logik wissenschaftlicher Forschungsprozesse: Das „Projektprinzip“ bindet die Forschungsfinanzierung an Anträge und Evaluierungen, an konkrete Vorhaben und deren zeitlich begrenzte Umsetzung (und lässt die Transaktionskosten explodieren).

Im paradoxer Weise ist die Soziologie von der Abhängigkeit von privaten bzw. privatwirtschaftlichen Drittmitteln weniger betroffen, da sich das Bestreben privater Geldgeber, sozialwissenschaftliche Projekte zu finanzieren, ohnehin in Grenzen hält. Bei der Soziologie kommt selten etwas heraus, mit dem man unmittelbar Geld verdienen kann. Natürlich kann man bei den bereits erwähnten Kleinprojekten die Interessen der Auftraggeber nicht völlig außer Acht lassen: Mit einer Patientenbefragung, in der sich das Krankenhaus durchwegs als Desaster erweist, wird man als Studienlieferant für die Zukunft nicht punkten können; aber solche Ergebnisse gibt es auch bei seriöser Wissenschaftlichkeit üblicherweise ohnehin nicht. Viel problematischer für die Soziologie (und andere Sozialwissenschaften) sind zwei andere Aspekte. Erstens bedeutet die Orientierung auf wirtschaftliche Verwertbarkeit, dass für die Sozialwissenschaften weniger Geld zur Verfügung steht. Sie sind schon immer die ,ärmeren Verwandten“ im Vergleich zu Naturwissenschaften und Technik, zu Biologie und Medizin gewesen; aber durch die forcierte Utilitarisierung ergibt sich ein noch stärkerer Trend, die Ressourcen in die „,brauchbaren“ Diszi-

plinen zu kanalisieren. Zweitens ist es die hegemoniale Gestalt naturwissenschaftlicher Projektförmigkeit, die von der Politik und von Förderinstitutionen als grundsätzlich gültiges Modell für 
jede Art wissenschaftlicher Arbeit genommen wird. Sie ist zwar für die meisten sozialwissenschaftlichen Projekte sinnlos, in manchen Fällen sogar schädlich, aber sie gilt als best practice. Solche wissenschaftlichen Moden sind oft auf ziemlich lange Zeit resistent gegen praktische Argumente und empirische Evidenz. Es handelt sich um Praktiken, die keiner Begründung oder Legitimierung bedürfen - „man macht das so“, wenn man auf der Höhe der Zeit sein will. Dass dabei mögliche Leistungen der Sozialwissenschaften verspielt werden, wird für die als „richtig“ angesehenen Praktiken noch lange nicht wirksam werden.

Preglau beklagt in einer zugehörigen These die sozialwissenschaftliche Innenschau:

„Die Praxis der Evaluation und der ,indikatorengesteuerten Ressourcenzuwendung' an den reformierten Universitäten erzwingt die Ausrichtung der Forschungstätigkeit der ForscherInnen an wissenschaftsinternen Evaluierungskriterien, in denen ,Wissenstransfer' und ,Praxisrelevanz' ebenfalls eine nur untergeordnete Rolle spielen.“

Der Vorwurf zielt gewissermaßen in die Gegenrichtung: Es seien nicht äußerliche (privatwirtschaftliche) Relevanzkriterien, die für eine Projektfinanzierung wichtig seien, sondern innerwissenschaftliche Kriterien, die jeden Praxisbezug vermissen lassen. Vermutlich handelt es sich jedoch um einen anderen Praxisbezug als jenen, der in der These von der Dominanz der Finanzierungsgeber angesprochen wird. Gemeint ist offensichtlich ein innerwissenschaftlich determinierter Praxisbezug, der auch kritische Analysen zulässt, im Unterschied zum extern determinierten Praxisbezug, der einfach praktische Verwertbarkeit im Auge hat. Insoweit hat Preglau auch wieder recht: Man hat nicht den Eindruck, dass alle kulturwissenschaftlichen Studien einen wesentlichen Beitrag zum Selbstverständnis einer spätmodernen Gesellschaft zu leisten imstande sind.

Es dürfte so sein, dass beide Varianten gleichzeitig vorkommen: die Einschränkung bestimmter Praxisrelevanz durch Auftraggeberlogik und die Einschränkung bestimmter Praxisrelevanz durch innerwissenschaftliche Spielerei. Auf der einen Seite gibt es einen Projektbetrieb, der mit Auftraggebern zurecht kommen muss, die ihrerseits unter zunehmendem Rechtfertigungsdruck stehen. Aber das ist eher der Bereich „kleiner Projekte“ im Bereich der angewandten Soziologie. Auf der anderen Seite gibt es starke Tendenzen in der Soziologie, sich tatsächlich in einen „spielerischen“ Bereich hinein zu bewegen, der jede praktische Relevanz für das Leben der Menschen vermissen lässt; der Anteil an „Glasperlenspielen“ im Rahmen der Soziologie hat zugenommen. Über Jahrzehnte hat sich die Soziologie beispielsweise so gut wie gar nicht mit Wirtschaftssoziologie beschäftigt (bzw. sie hat unter Wirtschaftssoziologie bloß die herkömmlichen Themen von Armut und Ungleichheit, allenfalls auch Wohlfahrtsstaat und Sozialpolitik verstanden); da war es nicht verwunderlich, dass die Ökonomen das gesamte Feld beherrschten und die Soziologen zur großen Wirtschaftskrise ebenso wenig zu sagen hatten wie die Volkswirte. Erst langsam beginnt sich ein wirtschaftssoziologisches Leben wieder zu regen, das mit 
ernsthafter Analyse statt mit pauschaler Systemkritik zu tun hat. Aber die Frage nach der Platzierung der Soziologie zwischen „Auftragslieferung“ und „Glasperlenspiel“ bleibt interessant.

\section{Aufklärungsverzichts-These}

Eine vierte These Preglaus schließt an die Projektlogik an: „Der Systemwandel der Universität von der öffentlichen Anstalt im Interesse der individuellen und gesellschaftlichen Emanzipation durch Bildung durch Wissenschaft, die von der Humboldtschen Universitätsreform an bis zur demokratischen Hochschulreform der 1970er Jahre Leitbildcharakter hatte, zum quasi-privaten ,Forschungsunternehmen' hat neben der inneren universitären Demokratie auch die Funktion der gesellschaftlichen Aufklärung an den Rand gedrängt: Die Universitäten treten nicht mehr als ,Sprachrohr stummen Leidens` und als ,Anwälte der Schwachen` öffentlich in Erscheinung und schreiten der Gesellschaft nicht mehr, mit der Fackel voran' (Ernst Bloch); statt dessen betreiben sie mehr oder weniger professionelles ,Public Relations' à la ,Lange Nacht der Forschung', in der es darum geht, mit mehr oder weniger spektakulären Show-Auftritten um die Gunst des Publikums zu werben, das ja mit seinen Steuern für den Wissenschaftsbetrieb aufkommt.“

Dieser Vorwurf apostrophiert die fundamentale Identität einer Universität. Und es ist richtig, dass Kategorien wie Emanzipation, Bildung durch Wissenschaft, Unterfütterung demokratischen Bewusstseins, Reflexivität und Urteilsfähigkeit durch akademische Kompetenz und derlei Hinweise mehr als Elemente des gegenwärtigen Diskurses über Universitäten praktisch verschwunden sind. Dass eine solche Orientierung mehr oder minder gleichgesetzt wird mit dem Engagement für Leiden und Schwäche, mit der wegweisenden „Fackel“, scheint mir weniger überzeugend. Marienthal war auch nicht ein Fanal, ein Aufruf, eine Führungsanmaßung, sondern solide Wissenschaft. Es wurde dargestellt, wie es war. Marienthal war weder Parteiprogramm noch Revolutionsaufruf. Zudem habe ich nicht den Eindruck, dass die meisten Sozialwissenschaftlerinnen und Sozialwissenschaftler, die ich kenne, zu politischen Führungsgestalten berufen wären. „Aufklärung“ liegt tatsächlich nicht unbedingt an der Spitze universitärer Selbstbeschreibungen, aber sie geschieht anders als durch Fackelzüge.

Preglau hat den Eindruck, dass die kritische Praxisrelevanz auch durch die vorherrschende Methodik eingeschränkt wird: „Ein ,positivistisches' Methodenideal weist eher in Richtung Neutralität / Distanz vom Forschungsobjekt und Abstinenz der ForscherInnen.“ Man kann nun allerdings darüber diskutieren, ob es nicht gerade zu den Stärken der Marienthal-Studie gehört, dass sie im Grunde weitgehend einem positivistischen Methodenideal folgt. Es wird (mit vielfältigen Methoden) geschildert, wie das Leben ist, ganz nüchtern, ganz einfach. (Natürlich wissen wir, dass die reine Objektivität immer ein Ideal ist, das niemals erreichbar ist, dass es vielmehr immer wesentliche „konstruktive“ Elemente gibt.) Es gibt natürlich weite Bereiche der Soziologie, in denen mit immer weiter verfeinerten Methoden triviale empirische Ergebnisse 
produziert werden, die einer soliden Interpretation ermangeln - und solche Arbeiten erscheinen in den besten Publikationsorganen. Umgekehrt sind weite Bereiche der Soziologie von positivistischen Empfehlungen ziemlich unbeeindruckt, es gibt nicht so selten massive ideologische Proklamationen, von den feministischen bis zu den postkolonialen Ansätzen und überhaupt in weiten Bereichen kulturforscherischer Bemühungen. Eine wirkliche positivistische Einengung, im Sinne einer kruden empirisch-statistischen Kausalmodellierung nach dem Vorbild der Naturwissenschaften des 19. Jahrhunderts, wäre natürlich tatsächlich katastrophal, aber sie wird nur von wenigen Vertretern des Faches verlangt, die von der gesellschaftlichen Wirklichkeit weit entfernt agieren. Ihnen sind auch Autoren wie Dahrendorf und Giddens, Bourdieu und Beck, Joas und Gross ein Dorn im Auge. Freilich wäre ein bisschen mehr „Intellektualität“ in der soziologischen Szene auch kein Fehler.

Die Fackel-Vorstellung entspricht nun allerdings der Engagiertheitssoziologie Michael Burawoys, der in seinem bekannten Plädoyer für eine Öffentlichkeitsorientierung der Soziologie neben der professionellen Soziologie, der Politikberatung und der kritischen Soziologie die Kategorie der public sociology so verstanden hat, dass hierbei das Bekenntnis für soziale Gerechtigkeit, politische Gleichheit, zwischenmenschliche Anerkennung und persönliche Emanzipation tragend sein soll (Burawoy 2005). Das ist freilich nicht jene öffentliche „Nachfrage“ nach soziologischer Information und Interpretation, der ich in zahlreichen Vorträgen und Interviews in der sozialen Wirklichkeit begegne. Die Öffentlichkeit will keine geistigen Sozialarbeiter und Polit-Coaches, keine Comtesche Priesterherrschaft, sie will in erster Linie wissen, was los ist - da draußen in dieser unübersichtlichen Gesellschaft im allgemeinen oder in Bezug auf spezifische Entwicklungen in bestimmten Lebensbereichen (Bude 2005). Das ist auch mit klassischen Aufklärungsideen verträglich: Durchblick bekommen, Reflexivität erhöhen, Orientierung ermöglichen. Dabei ist auch Urteilskraft gefragt.

Dass allerdings die Annäherung der Universität an die Gesellschaft nicht unter diesen Gesichtspunkten steht, sondern, wie Max Preglau mit Recht sagt, unter Marketing-Gesichtspunkten geschieht, ist nicht zu leugnen (Kieser 2010). Es hat sich ein umfangreiches Instrumentarium entwickelt, welches „unternehmerische Universitäten“ (Maasen und Weingart 2006) in ihrem Portefeuille haben müssen, wenn sie in ihren Leistungsvereinbarungen und Evaluierungen gut aussteigen wollen. Dazu gehört die Betreuung von Kindern, Älteren, Behinderten, die Obsorge für ökologische und ethnische Anliegen, die Wissenschaftsvermittlung durch alle möglichen Spektakel, Philosophieren im Kindergarten und Politisieren im Beisel, Weiterbildung und Didaktik für alle, Schreibnächte und offene Türen, Qualitätsmanagement und Forschungsmanagement, irgendetwas Künstlerisches und irgendetwas Kooperatives, viel darstellbar Internationales und imponierend Vernetztes - und was es halt sonst noch so gibt. Das hat Logik: Die Leistung einer Autofirma kann leicht besichtigt werden, ebenso wie die Leistung eines Supermarktes oder eines Friseurs; die Universität tut sich mit der Darstellung ihrer Leistung „Wissenschaft“ schwer. Wenn aber die Kernleistungen einer Organisation nicht darstellbar sind, weichen Orga- 
nisationen auf Sekundärleistungen aus. Im Falle eines Krankenhauses sind es schöne Aufenthaltsräume, Menüauswahl, Kinderbetreuungsprogramme und dergleichen; im Falle der Universität sind es Events, die für jeden, der mit Wissenschaft nicht viel anfangen kann, verstehbar sind (Perrow 1961). Das Repertoire solcher Events entwickelt sich im Konkurrenzkampf der Universitäten zu einem verpflichtenden Basisprogramm, mittels dessen die Selbstdarstellung in ständiger Abfolge allgemein verständlich erfolgt, auch wenn dadurch zur gleichen Zeit verstellt wird, was denn Wissenschaft eigentlich sei; mit dem fatalen Nebeneffekt, dass der budgetäre Inszenierungsaufwand anteilsmäßig steigen muss.

\section{Schlussbemerkung}

Es ist vielleicht eine allzu pessimistische Sicht, wenn Max Preglau meint, die Soziologie wäre nicht in der Lage, eine Studie wie jene von Marienthal heute durchzuführen; es gibt schon verschiedentlich (auch im deutschsprachigen, stärker noch im englischsprachigen Raum) beeindruckende Bücher und Aufsätze. Aber die Belebung und Normalisierung der Soziologie wird auch konterkariert durch Entwicklungen, die einen wesentlichen „Spielraum nach oben“ (zu einer hochwertigen, interessanten, praxisrelevanten Soziologie) als plausibel erscheinen lassen. Marienthal (Müller 2008) ist mit Recht ein Klassiker geworden.

\section{Literaturverzeichnis}

Bude, Heinz. 2005. Kommentar zu Michael Burawoy: Auf der Suche nach einer öffentlichen Soziologie. Soziale Welt 56 (4): 375-380.

Burawoy, Michael. 2005. For Public Sociology. Soziale Welt 56 (4): 347-374.

Habermas, Jürgen. 1988. Theorie des kommunikativen Handelns. Frankfurt a.M.: Suhrkamp.

Jahoda, Marie, Paul F. Lazarsfeld und Hans Zeisel. 1990. Die Arbeitslosen von Marienthal. Ein soziographischer Versuch über die Wirkungen langandauernder Arbeitslosigkeit. 8. Aufl. Frankfurt am Main: Suhrkamp.

Kieser, Alfred. 2010. Unternehmen Wissenschaft? Leviathan 38: 347-367.

Maasen, Sabine und Peter Weingart. 2006. Unternehmerische Universität und neue Wissenschaftskultur. die hochschule (1): 19-45.

Müller, Reinhard. 2008. Marienthal. Das Dorf, die Arbeitslosen, die Studie. Innsbruck: Studien-Verlag.

Perrow, Charles. 1961. Organizational Prestige. Some Functions and Dysfunctions. American Journal of Sociology 66 (4): 335-341.

Preglau, Max. 2008. „Die Arbeitslosen von Marienthal“ - 1. Warum ist diese Studie bis heute gesellschaftlich relevant? - 2. Wäre die Soziologie heute noch in der Lage, solch eine Studie durchzuführen? 
Auszug aus einem Referat, gehalten in Innsbruck am 10. November 2008. http://agso.uni-graz.at/marienthal/bibliothek/preglau_max_2008/1.htm

Scheler, Max. 1968. Die Formen des Wissens und die Bildung. (erstmals 1925). In: Philosophische Weltanschauung. 3. durchges. Aufl., hsrg. Max Scheler, 16-68. Bern u. a.: Francke.

Schelsky, Helmut. 1979. Auf der Suche nach Wirklichkeit. Gesammelte Aufsätze zur Soziologie der Bundesrepublik. München: Goldmann.

Manfred Prisching, geboren 1950 in Bruck/Mur, Studium der Volkswirtschaftslehre und der Rechtswissenschaften, Habilitation in Soziologie, Professor am Institut für Soziologie an der Universität Graz, Korrespondierendes Mitglied der Österreichischen Akademie der Wissenschaften, Mitglied des Österreichischen Wissenschaftsrates. 